\title{
Controlling anal incontinence in women by performing anal exercises with biofeedback or loperamide (CAPABLe) trial: Design and methods is
}

\author{
J. Eric Jelovsek ${ }^{\mathrm{a}, *}$, Alayne D. Markland ${ }^{\mathrm{b}}$, William E. Whitehead ${ }^{\mathrm{c}}$, Matthew D. Barber ${ }^{\mathrm{a}}$, Diane K. Newman ${ }^{\mathrm{d}}$, \\ Rebecca G. Rogers ${ }^{\mathrm{e}}$, Keisha Dyer ${ }^{\mathrm{f}}$, Anthony Visco ${ }^{\mathrm{g}}$, Vivian W. Sung ${ }^{\mathrm{h}}$, Gary Sutkin ${ }^{\mathrm{i}}$, Susan F. Meikle ${ }^{\mathrm{j}}$, \\ Marie G. Gantz ${ }^{\mathrm{k}}$, on behalf of the Pelvic Floor Disorders Network
}

a Obstetrics, Gynecology \&' Women's Health Institute, Cleveland Clinic, Cleveland, OH, United States

b Department of Medicine, University of Alabama at Birmingham, Birmingham, AL, United States

c Department of Gastroenterology, University of North Carolina at Chapel Hill, Chapel Hill, NC, United States

d Division of Urology, Department of Surgery, University of Pennsylvania, Philadelphia, PA, United States

e Departments of Obstetrics and Gynecology and Surgery, University of New Mexico Health Sciences Center, Albuquerque, NM, United States

${ }^{\mathrm{f}}$ Department of Obstetrics and Gynecology Kaiser Permanente, San Diego, CA, United States

g Department of Obstetrics and Gynecology, Duke University Medical Center, Durham, NC, United States

${ }^{\text {h }}$ Department of Obstetrics and Gynecology, Alpert Medical School of Brown University, Providence, RI, United States

${ }^{i}$ Department of Obstetrics, Gynecology and Reproductive Sciences, University of Pittsburgh Medical Center, Pittsburgh, PA, United States

${ }^{\mathrm{j}}$ The Eunice Kennedy Shriver National Institute of Child Health and Human Development, National Institutes of Health, Bethesda, MD, United States

${ }^{\mathrm{k}}$ RTI International, Research Triangle Park, NC, United States

\section{A R T I C L E I N F O}

\section{Article history:}

Received 28 May 2015

Received in revised form 10 August 2015

Accepted 12 August 2015

Available online 18 August 2015

\section{Keywords:}

Fecal incontinence

Randomized placebo controlled trial

Loperamide

Manometry-assisted biofeedback

Anal sphincter exercises

Factorial design

\begin{abstract}
A B S T R A C T
The goals of this trial are to determine the efficacy and safety of two treatments for women experiencing fecal incontinence. First, we aim to compare the use of loperamide to placebo and second, to compare the use of anal sphincter exercises with biofeedback to usual care. The primary outcome is the change from baseline in the St. Mark's (Vaizey) Score 24 weeks after treatment initiation. As a Pelvic Floor Disorders Network (PFDN) trial, subjects are enrolling from eight PFDN clinical centers across the United States. A centralized data coordinating center supervises data collection and analysis. These two first-line treatments for fecal incontinence are being investigated simultaneously using a two-by-two randomized factorial design: a medication intervention (loperamide versus placebo) and a pelvic floor strength and sensory training intervention (anal sphincter exercises with manometry-assisted biofeedback versus usual care using an educational pamphlet). Interventionists providing the anal sphincter exercise training with biofeedback have received standardized training and assessment. Symptom severity, diary, standardized anorectal manometry and health-related quality of life outcomes are assessed using validated instruments administered by researchers masked to randomized interventions. Cost effectiveness analyses will be performed using prospectively collected data on care costs and resource utilization. This article describes the rationale and design of this randomized trial, focusing on specific research concepts of interest to researchers in the field of female pelvic floor disorders and all other providers who care for patients with fecal incontinence.
\end{abstract}

(c) 2015 Elsevier Inc. All rights reserved.

\section{Introduction}

Fecal incontinence (FI) is a common problem with a prevalence ranging from $2-15 \%$ in community settings and up to $19 \%$ in women presenting to specialty clinics [1-3]. The age-adjusted prevalence of FI

\footnotetext{
is Supported by grants from the Eunice Kennedy Shriver National Institute of Child Health and Human Development (U10 HD054215, U10 HD041261, U10 HD041261, U10 HD054214, U10 HD041267, U10 HD069025, U10 HD069010, U10 HD069006, U01 HD069031) and the National Institutes of Health Office of Research on Women's Health.

* Corresponding author at: Cleveland Clinic, 9500 Euclid Ave. A81, Cleveland, OH 44195, United States.

E-mail address: jelovsj@ccf.org (J. Eric Jelovsek).
}

in non-institutionalized U.S. adults is $8.3 \%$ and consists of liquid stool in $6.2 \%$, solid stool in $1.6 \%$ and mucus in $3.1 \%$ [4].

Treatments for FI may be conservative including dietary manipulation, drug therapy, or behavioral treatments such as anal sphincter exercises/pelvic floor muscle training with or without biofeedback [5]. Surgical interventions include sphincter repair, rectocele repair, sacral neuromodulation, neosphincter, bulking injections and diversion [5]. Restoring normal bowel consistency is the first-line approach to therapy in patients with extremes of stool consistency (i.e. watery stools or hard and lumpy stools). This is usually accomplished using anti-diarrheal medications for patients with loose stools and laxatives or enemas for patients with constipation or incomplete emptying of the rectum. Since a significant proportion of women with FI will complain of leaking 
only with loose stool, an anti-diarrhea medication such as loperamide is often used as a first-line treatment. However, clinical evidence suggests that loperamide may have properties that could improve FI in patients with normal stool consistency [6]. A second conservative approach to treatment of women with FI is using anal sphincter exercises (pelvic floor muscle training) that may or may not include biofeedback assistance. Biofeedback therapy is often combined with additional behavioral interventions, such as bowel control strategies with sensory components. Multiple uncontrolled studies have suggested a benefit of behavioral interventions that include anal sphincter exercises with biofeedback for mixed liquid and solid stool consistency types of FI [7].

Since medical and behavioral approaches are often recommended as first-line treatments, [7] there is clinical value in determining the efficacy of these two conservative treatment options compared to placebo and basic educational information that are considered to be usual care for FI. Additionally, it is important to know if combined therapy offers any benefit over loperamide or anal sphincter exercises alone. The primary aims of the Controlling Anal incontinence by Performing Anal Exercises with Biofeedback or Loperamide (CAPABLe) trial are to compare the change from baseline in patient-reported scores of FI severity using the St. Mark's (Vaizey) Score [8] at 24 weeks after treatment initiation among: 1) women randomized to loperamide versus oral placebo for the treatment of $\mathrm{FI}$; 2) women randomized to anal sphincter exercises with biofeedback versus women randomized to usual care using a standardized educational pamphlet; and 3) women receiving the 2 active treatments together versus either active treatment alone. The purpose of this article is to describe the rationale and design of this randomized trial, focusing on specific research concepts of interest to researchers caring for women with FI, and to inform investigators designing randomized trials that combine medical interventions with standardized methods of behavioral interventions.

\section{Methods}

The Pelvic Floor Disorders Network (PFDN) is a clinical trials network composed of eight geographically diverse clinical centers in the United States, a Data Coordinating Center (DCC), and a Eunice Kennedy Shriver National Institute of Child Health and Human Development NICHD representative. The primary goal of the PFDN is to improve the level of knowledge about pelvic floor disorders and their treatments including pelvic organ prolapse, urinary incontinence and FI in women.

\subsection{Rationale for using a factorial design}

The value of using a factorial design for the CAPABLe study is that it allows a comparison of two first-line treatment options to placebo/ usual care in a single population, thereby improving efficiency, reducing trial cost and allowing a comparison of combination therapy compared to single therapy for FI in women. A factorial design is particularly valuable in evaluating a combination of interventions that have separate mechanisms of action. Our study design directly compares anal sphincter exercises with biofeedback versus usual care and drug therapy with loperamide versus placebo, as well as comparing both treatments together compared with either alone.

Anal sphincter exercises with biofeedback and drug therapy for FI have different mechanisms of action that culminate in improving sphincter strength and anorectal sensation. The most well accepted properties of loperamide are that it affects gastrointestinal smooth muscle by inhibiting intestinal peristalsis [9-11] increasing oral-cecal transit time [12] and increasing the mucosal exposure to intestinal effluent and decreasing stool weight [13]. Less known properties of loperamide are that it increases sensitivity of the recto-anal inhibitory reflex, [12] increases rectal perception and first incontinence volume [14] and increases anal sphincter squeeze duration [14]. These properties, along with decreasing fecal urgency, [14] promote quicker restroom-seeking behavior. Combined, this may serve as a barrier to stool leakage, and to reduce the volume of stool in the rectum [14]. Anal sphincter exercises with biofeedback increase the squeeze strength of the anal sphincter through strength training, and when combined with sensory training, may reduce sensory thresholds for patients with rectal hyposensitivity leading to passive fecal incontinence, and increase sensory thresholds for urgency in patients with urge-related fecal incontinence (hypersensitivity) [6].

\subsection{Design overview}

The CAPABLe study design is shown in Fig. 1. Eligible participants are randomized to one of four groups in a two-by-two factorial design: 1) usual care with oral placebo, 2) oral loperamide at a minimum dose of $2 \mathrm{mg}$ taken orally every other day to a maximum of $8 \mathrm{mg}$ daily with usual care, 3 ) anal sphincter exercise training with anorectal manometry-assisted biofeedback with usual care plus oral placebo and 4) combination oral loperamide with anal sphincter exercise training with manometry-assisted biofeedback with usual care. All participants receive an educational pamphlet developed by expert consensus which is publicly available from the National Institute of Diabetes and Digestive and Kidney Diseases (NIDDK) (http://bowelcontrol.nih.gov/ ). This pamphlet includes basic information about FI and available treatments such as behavioral techniques and medications. For this trial, the pamphlet was modified by removing a single reference in the document to the drug loperamide. We felt that this basic information that is publicly available should be provided to all patients seeking care for FI. Enrollment began April, 2014 and recruitment is anticipated to continue through March 2016 (clinicaltrial.gov \# NCT02008565).

\subsection{Study population}

The study population consists of adult women with at least monthly FI over the last 3 months that is bothersome enough to seek and desire treatment. Since FI may be a secondary symptom of colorectal malignancy, all women must have negative colon cancer screening based on one of the 2008 US Preventative Task Force recommended approaches. Women with predominant extremes of stool consistency on the Bristol Stool Form are excluded since patients with constipation are not candidates for potentially constipating agents such as loperamide and patients with chronic watery diarrhea may have a variety of causes for their diarrhea that need to be treated such as infectious etiologies [15]. A detailed list of the inclusion and exclusion criteria is in Table 1.

\subsection{Randomization and baseline measures}

Participants undergo a single randomization to one of the four treatment combinations. Randomization is a $0.5: 1: 1: 1$ allocation, to minimize the women who are randomized to no active therapy. Randomization is stratified by site using randomly permuted blocks; the sizes of the blocks are known only to the DCC.

After all screening assessments and consent are completed, the coordinator randomizes the participant at the baseline visit. Randomization is accomplished through the web-based data management system that assigns a randomization number that links to the biofeedback/usual care intervention assignment and loperamide/placebo treatment assignment. Women randomized to usual care undergo anal manometry measurements at baseline, 12 and 24 weeks. Women randomized to the anal manometry plus anal sphincter exercises with biofeedback intervention receive intervention at the baseline visit (following randomization) as well as at, 2, 4, 6, 9, 12 and 24 weeks. The loperamide/ placebo is stored in and dispensed from the investigational pharmacy at each clinical site. 


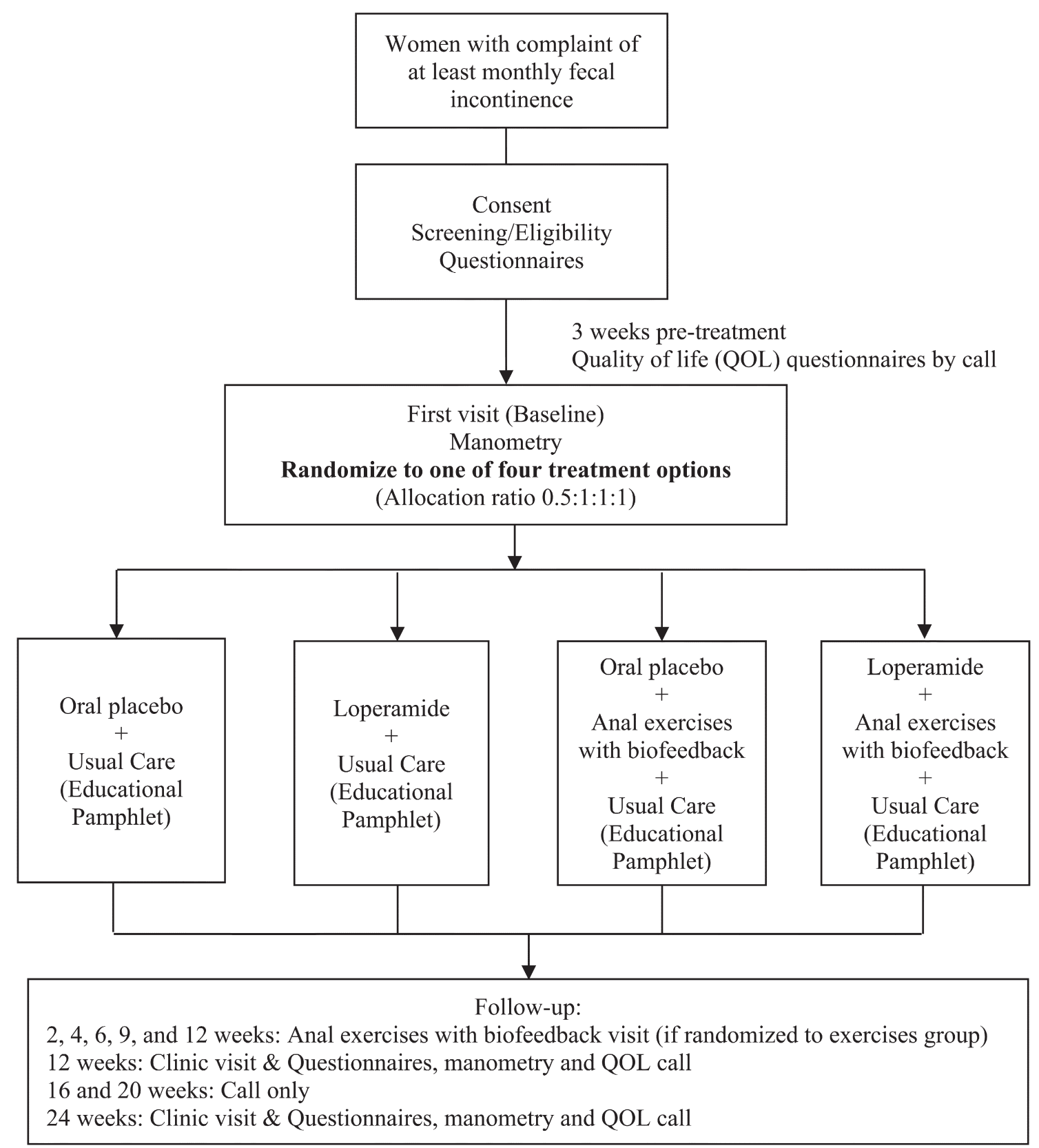

Fig. 1. Consort diagram for the study.

\subsection{Masking}

Table 2 demonstrates a summary of masking for the trial. For the anal sphincter exercises with biofeedback/usual care intervention, the physician, telephone interviewers, and the outcome evaluators are masked to the treatment assignment for the entire study duration. Behavioral interventionists are not masked to the anal sphincter exercises with biofeedback/usual care assignments, but are masked to the loperamide/placebo assignment. For the loperamide/placebo intervention, the physician, participant, study coordinator(s) and telephone interviewers are masked to the assignment for the duration of the study, and only the research pharmacist is unmasked. A placebo tablet was manufactured to match the loperamide tablet as closely as possible in appearance and weight. Loperamide and placebo tablets are over-encapsulation by standard DB Capsules (Capsugel, Greenwood, SC) which are backfilled with a standard inert excipient (Avicel).
Overencapsulation, bottling and labeling were done by a qualified contract manufacturing organization and drug supply provided to each site by the DCC. A two-part label was used such that an unmasked portion of the label can be removed and retained by the site pharmacist, and a masked label will remain on the bottle for distribution to the participant.

The protocol allows for dose adjustment during the trial ranging from $2 \mathrm{mg}$ loperamide/placebo (one capsule) orally every other day to a max of $8 \mathrm{mg}$ loperamide/placebo ( 4 capsules) daily. Dose adjustments are not performed by staff that collects outcome data since dose escalation or reduction may lead to unmasking; it was hypothesized that more placebo participants may dose escalate and more loperamide participants may dose reduce. Investigators felt it was important to allow for dose ranging due to efficacy and side-effects rather than limit participants to one or two escalation options. This allows for more accurate determination of an appropriate dose of loperamide for 
Table 1

Protocol inclusion and exclusion criteria.

\begin{tabular}{l}
\hline Inclusion criteria: \\
\hline 1. Age $\geq 18$ years \\
2. Fecal incontinence defined as any uncontrolled loss of liquid or solid fecal \\
material that occurs at least monthly over the last 3 months that is \\
bothersome enough to desire treatment \\
3. If a patient is 50-75 years old they should have current negative colon cancer \\
screening based on the US Preventative Task Force recommendation (2008) \\
that includes either: \\
a. Annual screening with high-sensitivity fecal occult blood testing or \\
b. Sigmoidoscopy every 5 years, with high-sensitivity fecal occult blood testing \\
every 3 years or \\
c. Screening colonoscopy every 10 years
\end{tabular}

Patients who are $\geq 76$ years old do not need routine colon cancer screening since the likelihood that detection and early intervention will yield a mortality benefit that declines after age 75 because of the long average time between adenoma development and cancer diagnosis. Patients 50-75 years old without a current negative colon cancer screening may elect to undergo one of these screening options and if results are normal, they may continue to screen for eligibility in the trial.

Exclusion criteria:

1. Stool consistency over the last 3 months that includes items 1 or 7 based on the Bristol Stool form scale

2. Current bloody diarrhea (loose, watery stools 3 or more times a day with blood)

3. Current or past diagnosis of colorectal or anal malignancy

4. Diagnosis of inflammatory bowel disease

5. Current or history of rectovaginal fistula or cloacal defect

6. Rectal prolapse (mucosal or full thickness)

7. Prior removal or diversion of any portion of colon or rectum

8. Prior pelvic floor or abdominal radiation

9. Refusal or inability to provide written consent

10. Inability to conduct telephone interviews conducted in English or Spanish

11. Fecal impaction by rectal and abdominal exam

12. Untreated pelvic organ prolapse beyond the hymen; patients with prolapse beyond the hymen who are currently using a pessary are eligible

13. Incontinence only to flatus

14. Has taken any loperamide (Imodium $\left.{ }^{\circledR}\right)$ or diphenoxylate plus atropine (Lomotil®) in the last 30 days

15. Previously received and failed treatment of fecal incontinence using loperamide (Imodium ${ }^{\circledR}$ ) or diphenoxylate plus atropine (Lomotil $®$ ) over the last 3 months

16. Current supervised anal sphincter exercise/pelvic floor muscle training with biofeedback

17. Previously received and failed treatment of fecal incontinence using supervised anal sphincter exercise/pelvic floor muscle training with biofeedback

18. Previous allergy or intolerance to loperamide

19. Pregnant, nursing, or planning to become pregnant before the end of the study follow-up period.

20. Childbirth within the last 3 months

21. Neurological disorders known to affect continence, including spinal cord

injury, advanced multiple sclerosis or Parkinson's disease and debilitating stroke

22. Known diagnosis of hepatic impairment

23. Chronic abdominal pain in the absence of diarrhea

24. Acquired Immunodeficiency Syndrome (AIDS)

25. Currently taking anti-retroviral drugs

patients with FI. To accommodate this dose adjustment and minimize the possibility of the coordinator influencing dose, adjustments are standardized using a dose escalation/reduction matrix driven by the participant's rating of global symptom control and tolerability; this matrix is outlined in Table 3.

\subsection{Medical intervention with loperamide versus placebo}

Participants randomized to the loperamide treatment group begin with $2 \mathrm{mg}$ of loperamide per day. The FDA approved initial dose in adults with acute or chronic diarrhea is $4 \mathrm{mg}$ (two capsules) followed by $2 \mathrm{mg}$ (one capsule) after each unformed stool until diarrhea is
Table 2

Masking summary.

\begin{tabular}{|c|c|c|}
\hline & $\begin{array}{l}\text { Masked to } \\
\text { loperamide/placebo } \\
\text { Intervention }\end{array}$ & $\begin{array}{l}\text { Masked to anal sphincter exercises with } \\
\text { biofeedback/usual care intervention }\end{array}$ \\
\hline Participant & Yes & No \\
\hline \multirow{3}{*}{$\begin{array}{l}\text { Study } \\
\text { coordinator }\end{array}$} & Yes & Yes \\
\hline & & $\begin{array}{l}\text { - Primary outcome } \\
\text { - Most secondary outcomes } \\
\text { No }\end{array}$ \\
\hline & & $\begin{array}{l}\text { - Anal sphincter exercises with } \\
\text { biofeedback/usual care treatment } \\
\text { assignment } \\
\text { - Adverse events } \\
\text { - Medical follow-up } \\
\text { - Adherence } \\
\text { - Productivity data collection }\end{array}$ \\
\hline $\begin{array}{l}\text { Telephone } \\
\text { interviewer* }\end{array}$ & Yes & Yes \\
\hline Study physician & Yes & Yes \\
\hline Evaluator $^{* *}$ & Yes & Yes \\
\hline Interventionist ${ }^{\#}$ & Yes & No \\
\hline Pharmacist & No & Yes \\
\hline
\end{tabular}

* Telephone interviewer: individual from the Quality of Life Call Center.

** Evaluator: the individual(s) at the clinical sites performing outcome assessments,

\# Interventionist: the individual(s) at the clinical sites providing the anal sphincter exercises with biofeedback/ intervention and conducting rectal manometry measurements.

controlled, after which, the dosage should be titrated to meet individual requirements, up to $8 \mathrm{mg} /$ day. For this study, participants are seen in person at their first treatment visit, 12 weeks, and 24 weeks. Study coordinators also call the participants at the 2, 6, 16 and 20 week intervals to administer global instruments to assess efficacy and tolerability of loperamide/placebo and manage dose adjustments accordingly. For evaluation of patient perceived efficacy, the Patient Global Symptom Control rating scale (PGSC) is used [20]. Dose escalation is based exclusively on the result of the PGSC as shown in the Table 3, ranging from 1 (strongly disagree) to 5 (strongly agree). The participant is instructed to either maintain the current drug/dose if PGSC is 4 or 5 (agree/strongly agree that their treatment is giving adequate control of stool leakage), or dose escalate if PGSC is 1-3 (disagree/strongly disagree that their treatment is giving adequate control) in the absence of bothersome side effects. Participants who report inadequate control of stool leakage on the PGSC are instructed to increase the daily dose of study medication by one capsule ( $2 \mathrm{mg}$ loperamide or placebo) up to a maximum of 4 capsules ( $8 \mathrm{mg}$ loperamide or placebo) per day. Participants are not required to take the entire daily dose at one time.

Bothersome side effects are monitored using a patient global tolerability scale (PGTS) which is modified from the PGSC [20]. Dose reduction is based on the participants' responses to the PGTS. The participant is instructed to reduce the current drug/dose if PGTS is 4 or 5 (agree/ strongly agree that the treatment is giving them bothersome side effects) as shown in the Table 3. Participants who report bothersome side effects of the medication are instructed to decrease the daily dose of study medication by one capsule to a minimum of one capsule ( $2 \mathrm{mg}$ loperamide or placebo) every other day. They are instructed to discontinue the study medication if they have a PGSC score of 1-3 (inadequate control of stool leakage) combined with a PGTS score of 4-5 (bothersome side effects). If bothersome side effects are reported at any time during the study, the participant is instructed to contact the clinical site and the site investigators may choose to discontinue or temporarily hold the study drug. In addition, dose reduction or medication discontinuation can occur at any time if a patient reports bothersome side effects. 
Table 3

Dose Escalation/change in study medication and management of adverse events between medication steps, based on efficacy and tolerability.

\begin{tabular}{|c|c|c|c|}
\hline & & \multicolumn{2}{|l|}{ Efficacy } \\
\hline & & Yes (PGSC 4,5) & No (PGSC 1-3) \\
\hline \multirow[t]{2}{*}{ Tolerability } & $\begin{array}{l}\text { Yes } \\
\text { (PGTS 1-3) }\end{array}$ & No change in medication & Dose escalate $^{*}$ \\
\hline & $\begin{array}{l}\text { No } \\
\text { (PGTS 4-5) }\end{array}$ & Dose reduction $^{* *}$ & Patients are instructed to discontinue medication \\
\hline
\end{tabular}

The Patient Global Symptom Control rating (PGSC) scale [20] is:

My current treatment is giving me adequate control of my stool leakage.

Disagree $\quad 1 \quad 2$

Strongly

4

4

Agree

The Patient Global Tolerability rating Scale (PGTS) is:

My current medication is giving me bothersome side effects

Disagree

4

Strongly

Strongly

* Max dose of 4 capsules ( $8 \mathrm{mg}$ loperamide or placebo) per day.

** Minimum dose of 1 capsule ( $2 \mathrm{mg}$ loperamide or placebo) every other day.

\subsection{Manometric biofeedback intervention versus usual care alone}

To address the limitations of most FI biofeedback intervention studies, investigators for this study developed 3 separate anal sphincter exercises with biofeedback sub-protocols: (1) strength training with home anal sphincter exercises, (2) sensory training for hyposensitive rectal distention, and (3) sensory training for urge-resistance rectal distention. Anorectal manometry (ARM) data were also standardized for this trial, and include measurements of strength, squeeze duration, and sensation. All participants have ARM performed at baseline, 12 weeks, and 24 weeks. Among participants randomized to anal sphincter exercises with biofeedback, the ARM data are key measures for determining which of the sensory sub-protocols to use along when combined with data from the patient-reported bowel diaries.

As provider experience is a major factor in performing anal sphincter exercises with biofeedback for FI, all study interventionists participating in the CAPABLe trial were required to review standardized online content, undergo hands-on training conducted with live models, and assessment by experienced providers trained using protocol-specific performance checklists. All interventionists were required to review online learning modules including instructional videos prior to attending the training sessions. Training included e-learning content provided using slides, problem-oriented patient case discussions, and hands-on practice with live models. Each interventionist completed a 75 minute certification exam in the conduct of patient visits that included performing ARM diagnostic testing, manometric biofeedback with strength and sensory training and education on when to use each sensory protocol. Trained interventionists include certified registered nurse practitioners, physician assistants and physical therapists. A minimum of two certified interventionists are available at each clinical site.

Subjects randomized to the anal sphincter exercises with biofeedback receive a structured individualized program during the first 12weeks of the 24-week study including visits at baseline, 2, 4, 6, 9, and 12 weeks for a total of 6 biofeedback intervention visits. Diagnostic ARM evaluation is performed at each visit in order to guide the manometric biofeedback protocols and home anal sphincter exercises. All participants receive the strength training sub-protocol focusing on correct anal sphincter muscle isolation, anal sphincter contraction strength, and the duration of the contraction beginning at the first visit and continuing on subsequent visits as needed. The visits at, 2, 4, 6 and 9 weeks include a sensory protocol for participants who have partially lost the ability to detect the presence of stool in the rectum (called hyposensitivity) or a urge resistance protocol for those participants who experience strong sensations of urgency to defecate which are difficult to suppress (called hypersensitivity). At each visit, interventionists provide feedback using standardized handouts given that reinforce these techniques for home use. A manual of procedures describes guidelines in an algorithm approach to determine the correct sensory subprotocol to use based on pre-determined criteria. We collaborated with the device manufacturer to develop on-screen prompts to guide the interventionists through these protocols. To help ensure adherence with this standardized regimen and treatment fidelity, audiotapes are obtained from select biofeedback sessions and members of the study team audit the tapes using standardized procedure checklists.

The software and catheter from the mcompass (Medspira, Minneapolis, $\mathrm{MN}$ ) manometry device was redesigned specifically for this protocol. Building on existing software available for ARM, the research team partnered with staff from Medspira to create novel biofeedback software specifically tailored for the 3 biofeedback subprotocols in the trial. The mcompass system uses a tablet computer which wirelessly connects to the catheter. This system allows for equipment mobility and visual feedback to the participant for the strength and sensory training. The system's manometric catheter simultaneously measures pressures in the rectum and the anal canal to help participants isolate sphincter contractions while avoiding inappropriate contractions of abdominal wall muscles during strength training. The catheter contains a balloon that is positioned in the rectum and used to simulate rectal filling for the sensory protocols. All participants randomized to the anal sphincter exercises with biofeedback protocol are also prescribed a home exercise program based on their individual performance during the intervention visits. Participants record their home exercises in an exercise record and complete a seven-day bowel diary before the next anal sphincter exercise with biofeedback visit.

Subjects randomized to usual care are scheduled for visits with the interventionist at baseline, 12 , and 24 weeks. ARM data are collected at these visits. The usual care subjects complete a 7-day bowel diary prior to these visits. At the baseline visit, the interventionists give the same structured education using the NIDDK bowel control handout that the anal sphincter exercises with biofeedback group receives.

\subsection{Data collection and follow up}

A timeline of visits, events and data collection is listed in Table 4. After initial screening, eligible patients will be given a 7-day bowel diary and receive a Quality of Life (QOL) telephone interview. Randomization, review of bowel diary, and baseline ARM are performed at the baseline visit scheduled 2 to 4 weeks after the screening visit.

We attempted, when possible, to keep the study visits similar between groups. However, the group randomized to receive anal sphincter exercises with biofeedback has more intervention visits by the nature of the treatment. Participants randomized to usual care have in-person visits at baseline, 12 and 24 weeks. Conversely, those 
randomized to anal sphincter exercises with biofeedback have inperson visits at baseline, 2, 4, 6, 9, 12 and 24 weeks. In our judgment, if we had kept the number of visits the same between the anal sphincter exercise with biofeedback group and the usual care group, this would have been perceived as artificial and burdensome to the usual care participants and may have influenced patient retention in the trial.

A supply of study drug (loperamide) or placebo is dispensed at the baseline and 12 week visits. At 12 and 24 weeks, the participants are asked to bring their recently completed bowel diary to the clinic, to complete QOL interviews by telephone, and to undergo repeat ARM evaluation. Participants receive a telephone call from the research coordinator at weeks 2, 6, 16 and 20 to assess updates in medical history, medications, efficacy and tolerability of medications. Adverse events are collected on all participants at the 2, 6, 12, 16, 20, and 24 week calls or visits and at any time in between when self-reported bothersome symptoms are noted.

Participants in the anal sphincter exercises with biofeedback group are instructed to record in a diary the use of exercises at home. Compliance with loperamide/placebo is monitored using pill counts, a single question provider-reported adherence [23], and the modified Medication Adherence Self-Report Inventory (MASRI) [24] at the 12 and 24 week in-person visits. These three methods of "triangulation" are consistent with recommendations by Osterberg to use several methods in order to improve accuracy in assessing medication adherence [25]. Cost data and resource utilization are collected at the screening visit and at the 12 and 24 week visits. The primary outcome is assessed at the 24 week in-person visit by a masked coordinator. At this final visit, participants are asked if they know whether they received loperamide or placebo (i.e., whether they became unmasked).

\subsection{Considerations in the selection of primary outcome}

To meet recommendations of the NIH consensus statement and the Cochrane review, the protocol committee focused on using a primary outcome measure that could incorporate the patient perspective as well as FI frequency, severity, bother, fecal urgency and patient desire for treatment. Furthermore, the team also desired an instrument that had published evidence supporting its validity and data to guide the interpretation and clinical relevance of improvement in scores from the patient perspective. The protocol committee considered a variety of patient-centered outcome measures including severity scales, FI episodes, quality of life scales, global satisfaction scales, overall treatment satisfaction as well as using multiple primary endpoints. Unfortunately, no single primary outcome available for trials in FI that is broadly accepted was available. A variety of measures that more or less met recommended requirements were reviewed, and the committee elected to use change from baseline to 24 weeks in the St. Mark's (Vaizey) FI severity scale as the primary outcome because the scale meets as many of the desired attributes as possible while maintaining participant acceptability. Data exist that correlate improvement in the frequency of FI episodes with improvement in the St. Mark's (Vaizey) Score $(\mathrm{r}=0.79, \mathrm{P}<.001)[8]$, as well as moderate correlation with changes in maximum incremental squeeze pressure $(\mathrm{r}=-0.30, \mathrm{P}<0.05)[26]$.

The St. Mark's Score is also proved responsive to change. When assessing the correlation between the St. Mark's Score and global impression of improvement after treatment, data demonstrate that average St. Mark's Score severity scores are 1 point lower than baseline for patients who rate their situation as "worse or equal", 4 points lower for patients who reported their situation to be "better", and 9 points

Table 4

Timeline of visits, events and data collection.

\begin{tabular}{|c|c|c|c|c|c|c|c|c|c|c|}
\hline Interventions and outcomes & ${ }^{\dagger}$ Screening visit & $\begin{array}{l}\text { First visit } \\
\text { (Baseline) }\end{array}$ & 2 weeks & 4 weeks & 6 weeks & 9 weeks & 12 weeks & $\begin{array}{l}16 \text { weeks } \\
\text { (Call only) }\end{array}$ & $\begin{array}{l}20 \text { weeks } \\
\text { (Call only) }\end{array}$ & 24 weeks \\
\hline \multicolumn{11}{|l|}{ All participants: } \\
\hline Eligibility $^{*}$ and informed consent & $\mathrm{X}$ & & & & & & & & & \\
\hline Socio-demographics & $\mathrm{X}$ & & & & & & & & & \\
\hline Medical history & $\mathrm{X}$ & & & & & & & & & \\
\hline $\begin{array}{l}\text { Primary outcome } \\
\text { St. Mark's (Vaizey) Score [8] }\end{array}$ & $\mathrm{X}$ & & & & & & $\mathrm{X}$ & & & $\mathrm{X}$ \\
\hline Randomization & & $\mathrm{X}$ & & & & & & & & \\
\hline Loperamide/ placebo dispensing & & $\mathrm{X}$ & & & & & & & & \\
\hline Anal sphincter tone & $\mathrm{X}$ & & & & & & $\mathrm{X}$ & & & $\mathrm{X}$ \\
\hline 7-day bowel diary review ${ }^{* *}$ & & $\mathrm{X}$ & & & & & $\mathrm{X}$ & & & $\mathrm{X}$ \\
\hline Other measures ${ }^{* * *}$ & $\mathrm{X}$ & & & & & & $\mathrm{X}$ & & & $\mathrm{X}$ \\
\hline Cost/resources assessments & $\mathrm{X}$ & & & & & & $\mathrm{X}$ & & & $\mathrm{X}$ \\
\hline Adverse events & & & & & & & $\mathrm{X}$ & & & $\mathrm{X}$ \\
\hline Efficacy (PGSC) [20] & & & $\mathrm{X}$ & & $\mathrm{X}$ & & $\mathrm{X}$ & $\mathrm{X}$ & $\mathrm{X}$ & $\mathrm{X}$ \\
\hline Tolerability (PGTS) & & & $\mathrm{X}$ & & $\mathrm{X}$ & & $\mathrm{X}$ & $\mathrm{X}$ & $\mathrm{X}$ & $\mathrm{X}$ \\
\hline Adherence measures & & & & & & & $\mathrm{X}$ & & & $\mathrm{X}$ \\
\hline Exercise record & & & $\mathrm{X}$ & $\mathrm{X}$ & $\mathrm{X}$ & $\mathrm{X}$ & $\mathrm{X}$ & & $\mathrm{X}$ & $\mathrm{X}$ \\
\hline Quality of life measures ${ }^{* * * *}$ & $\mathrm{X}$ & & & & & & $\mathrm{X}$ & & & $\mathrm{X}$ \\
\hline \multicolumn{11}{|l|}{ Usual care group: } \\
\hline Diagnostic manometry & & $\mathrm{X}$ & & & & & $\mathrm{X}$ & & & $\mathrm{X}$ \\
\hline Bowel diary review & & $\mathrm{X}$ & & & & & $\mathrm{X}$ & & & $\mathrm{X}$ \\
\hline \multicolumn{11}{|l|}{ Anal exercises with manometry-assisted biofeedback group } \\
\hline Diagnostic manometry & & $\mathrm{X}$ & & & & & $\mathrm{X}$ & & & $\mathrm{X}$ \\
\hline Anal exercises with manometry-assisted biofeedback & & $\mathrm{X}$ & $\mathrm{X}$ & $\mathrm{X}$ & $\mathrm{X}$ & $\mathrm{X}$ & $\mathrm{X}$ & & & \\
\hline Bowel diary review & & $\mathrm{X}$ & $\mathrm{X}$ & $\mathrm{X}$ & $\mathrm{X}$ & $\mathrm{X}$ & $\mathrm{X}$ & & & $\mathrm{X}$ \\
\hline
\end{tabular}

Note: Baseline visit is 3 weeks after screening visit. All timelines have a window of $+/-1$ week.

$\dagger$ All screening activities may be done by either the masked or unmasked coordinator

* Eligibility includes provider's physical exam to assess applicable exclusion criteria.

** All participants will be instructed by the masked coordinator to collect data in the bowel diary for one week prior to the baseline, 12 week, and 24 week visits.

*** Other measures include: Bristol Stool Form scale [15], ABLe Measure, PISQ-IR extended version [32], Body Image Scale [33,34], Dietary Fruit/Veggie/Fiber Screener [35,36].

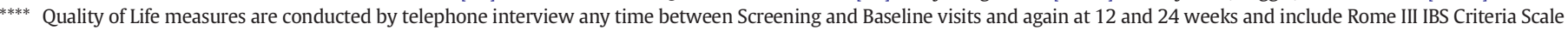

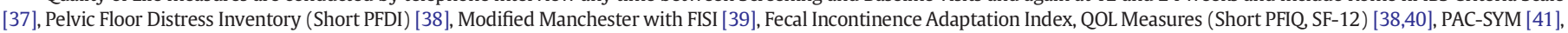
and Modified PGI-I [42]. 
lower in patients who rate their situation "much better" $(\mathrm{P}<.05)$. This also supports the assertion that the St. Mark's Score is consistent with patients' subjective perception of relief from FI [26]. For all of these reasons, the team felt that the St. Mark's Score is the best existing measure that captures a meaningful outcome of FI treatment.

\subsection{Secondary outcome measures}

The secondary outcome measures listed in Table 5 are alternative measures of treatment efficacy. The key secondary outcomes are the frequency of FI episodes on a 7-day bowel diary, and validated quality of life measures, which are measured at baseline, 12 weeks, and 24 weeks in all treatment groups. Additional secondary outcome measures are tallies of adverse events and estimates of the cost of delivering the interventions. Secondary safety outcomes include monitoring of adverse events. Comparisons of adverse events and serious adverse events between treatment groups will be reported at the end of the study. All adverse events and serious adverse events reported by participants are compared between treatment groups on a quarterly basis and reviewed by the Data Safety Monitoring Board. Possible adverse events include abdominal distension, abdominal pain or discomfort, allergic reactions, constipation, nausea, vomiting, tiredness, dizziness, and drowsiness. Bothersome adverse events will be classified using the PGTS. Study drug dose reduction will be based exclusively on the result of the PGTS.

\subsection{Measures of compliance with study interventions}

Adherence to taking the study medication, the exercise log, number of anal sphincter exercises and biofeedback visits completed, and audits of tape recordings to assess whether the interventionists adhered to the

\section{Table 5}

Secondary outcomes in CAPABLe.

Medication and Medical History Review

Previous and interval treatment for pelvic floor disorders or bowel disorders

7-day bowel diary

Anal sphincter tone on physical examination using the Digital Rectal Examination Scoring System

Manometry measures including: distance $(\mathrm{cm})$ of catheter insertion to locate high pressure zone (HPZ) of anal canal, resting anal canal pressures ( $\mathrm{mm}$ of $\mathrm{Hg}$ ) at 2 $\mathrm{cm}, 1 \mathrm{~cm}$, and $0 \mathrm{~cm}$ insertion, resting rectal pressures $(\mathrm{mm} \mathrm{Hg}$ ) with anal sensor at $2 \mathrm{~cm}, 1 \mathrm{~cm}$, and $0 \mathrm{~cm}$ insertion, maximum anal and rectal pressures during squeeze with the catheter at the HPZ, volume of air $(\mathrm{mL})$ at first sensation for perception of rectal distention, volume of air $(\mathrm{mL})$ at urge to defecate, maximum tolerable rectal volume of air $(\mathrm{mL})$, volume of air $(\mathrm{mL})$ at sensation of strong urge and rectal balloon pressure at sensation of strong urge

Dietary fiber intake using the Fruits/Vegetables/Fiber Screener questionnaire [36]

Pelvic Symptoms: Pelvic Floor Distress Inventory Short Form (PFDI-Short) including all subscales, Pelvic organ prolapse/urinary incontinence sexual function questionnaire - IUGA Revised (PISQ-IR) and an additional anal intercourse question Modified Manchester Health Questionnaire that includes the 4-item Fecal Incontinence Severity Index (FISI) [32,38,39]

Modified Patient Global Impression of Improvement (PGI-I) for bowel function, Adaptation using the Fecal Incontinence Adaptation Index, Defecatory symptoms as measured by Patient Assessment of Constipation Symptoms questionnaire (PAC-SYM) [41,42]

Quality of life - Medical Outcome Study Short-Form-12 (SF-12) including all subscales, Pelvic Floor Impact Questionnaire Short Form including all subscales (PFIQ-Short), Body Image Scale [33,38,40]

Efficacy and Tolerability using the Patient Global Symptom Control rating scale (PGSC) and Patient Global Tolerability Scale (PGTS) modified from the PGSC [20]

Compliance with treatment using pill counts, a single question provider-reported adherence [23] and the modified Medication Adherence Self-Report Inventory (MASRI) [24]

Adverse events

Productivity loss including: days of missed work, missed household chores, caregiver costs, travel time, transportation costs, out of pocket appointment costs, incontinence products costs and laundry costs.

Rome III IBS criteria [37]

Bowel leakage as measured by the Accidental Bowel Leakage (ABLe) instrument procedure manual are measures that address the degree of adherence with study interventions. The questionnaire to assess whether participants were unmasked to whether they received loperamide or placebo assesses the integrity of masking in the drug treatment arm.

\subsection{Mediators of treatment effects}

The ARM test at baseline, 12 and 24 weeks is a process measure that assesses whether the anal sphincter exercises and biofeedback are having the expected impact on anal canal squeeze pressure, rectal pressure during squeezing, and sensory thresholds for first sensation and strong urge. These are hypothesized to mediate the impact of pelvic floor exercises and biofeedback on the St. Mark's Score of FI severity. The ARM test is performed at baseline, 12, and 24 weeks. Sociodemographic variables measured at baseline will also be examined as possible moderators of treatment efficacy.

\subsection{Sample size and power}

Table 6 describes the hypothesized value of the primary outcome in each of the treatment groups. A difference in change from baseline in St. Mark's (Vaizey) Score at 24 weeks of at least 5 is hypothesized between each of the groups assigned to receive a single active treatment and those randomized to usual care (placebo and educational pamphlet), and a modest negative interaction is assumed in the combined therapy arm (loperamide and anal sphincter exercise + biofeedback). This is consistent with the minimally important difference (MID) of -5 for the St. Mark's (Vaizey) Score derived using three different methods of detecting a clinically important difference [27]. In the study conducted by Bols et al., various methods were used to estimate the MID and the authors concluded that an MID of -5 seemed preferable and yielded the lowest misclassification rate [27]. We also requested from Bols et al. a reanalysis of the MID of the St. Mark's (Vaizey) Score without the medication item in the questionnaire, and it appears that an MID of -5 is still appropriate for the modified St. Mark's (Vaizey) Score without medication.

Randomization will be unequal, with fewer patients randomized to the combination of placebo drug and usual care (0.5:1:1:1 allocation). Power calculations were based on the hypothesized changes from baseline in St. Mark's (Vaizey) Score at 24 weeks shown in Table 6. If we assume a follow up rate of $100 \%$ at 24 weeks, then a sample size of 245 , with 35 participants in the placebo/usual care group and 70 in each of the other three groups, will provide $90 \%$ power to detect a difference in each treatment arm (loperamide vs. placebo averaged over the two exercise treatments and exercise + biofeedback vs. usual care averaged over the two drug treatments) at a 0.025 level of significance. It will also provide $80 \%$ power to detect a difference in drug alone or exercise alone vs. the placebo/usual care combination, and 55\% power to detect a difference in combined drug and exercise vs. either intervention alone, at a 0.05 level of significance. The power to detect an interaction that is significant at the 0.05 or 0.10 levels will be approximately $14 \%$ and $23 \%$, respectively. Despite having only $55 \%$ power, we chose to include the third aim as primary rather than secondary, because we think it is important to compare combination therapy to each treatment alone. It may be particularly important if the magnitude of the interaction between the treatment arms is greater than we anticipate, in

\section{Table 6}

Hypothesized change from baseline in St. Mark's (Vaizey) Score at 24 weeks in each treatment group.

\begin{tabular}{lll}
\hline \multirow{2}{*}{ Drug } & Exercise & \\
\cline { 2 - 3 } & $\begin{array}{l}\text { Usual care } \\
\text { (educational pamphlet) }\end{array}$ & $\begin{array}{l}\text { Anal sphincter exercise }+ \\
\text { biofeedback }\end{array}$ \\
\hline Placebo & -2 & -7 \\
Loperamide & -7 & -10 \\
\hline
\end{tabular}


which case our power to detect the differences would also be higher than our estimates indicate. If we assume a conservative follow up rate of $85 \%$ at 24 weeks, and we use analysis methods that are consistent with an assumption that missing outcomes will be missing at random, then power calculations yield a sample size estimate of 294 patients (42 in the usual care (educational pamphlet)/placebo group and 84 in each of the other treatment combinations) to obtain the power levels described above.

\subsection{Data analysis plan}

The primary outcome, change from baseline in St. Mark's (Vaizey) Score at 24 weeks, will be compared among treatment groups using linear regression. Because the St. Mark's (Vaizey) Score is assessed at both 12 and 24 weeks, the primary analysis will be based on a longitudinal model, with changes from baseline in St. Mark's (Vaizey) Score at both 12 and 24 weeks as the dependent variable, and the independent variables including treatment assignment (both drug and exercise), interaction between drug and exercise treatment assignments, week (12 or 24), 2- and 3-way interactions between week and treatment assignments, Rome III irritable bowel syndrome (IBS) clinical trial status, interaction between Rome III IBS clinical trial status and treatment, and clinical site. If the interactions between treatment arms and/or between treatment and Rome III IBS status are not statistically significant, then the statistical test comparing the treatment groups will be averaged over the other variable involved in the interaction. If there is a statistically significant interaction, then the treatment groups will be compared within each level of the other variable involved in the interaction. The interactions between time and treatment arms will allow for statistical tests to compare the treatments at the 24 week time point for the primary outcome, since the model will include change from baseline at both 12 and 24 weeks. Patients with IBS may be included in the trial as IBS remains an important risk factor for fecal incontinence. However, the analysis will control for this using the interactions described. Under the assumption that any missing outcome data will be missing at random (thus, missing St. Mark's (Vaizey) Scores at 24 weeks may be related to both 12 -week outcomes and covariates), this model will produce more accurate estimates in the presence of missing data than one that models only outcomes at 24 weeks. The model will account for the dependence between repeated measurements on the same subject. Two-sided tests of the effect of drug treatment assignment (loperamide vs. placebo) and exercise treatment assignment (anal sphincter exercises with biofeedback vs. usual care (educational pamphlet)) on change from baseline in St. Mark's (Vaizey) Score at 24 weeks will be performed at a type I error level of 0.025 . The same model will be used to test whether there is a difference in combination treatment compared to loperamide and combination treatment compared to anal sphincter exercises with biofeedback in change from baseline in St. Mark's (Vaizey) Score at 24 weeks with type I error of 0.05 . We will also test for differences in the change in score over time among the 4 individual treatment groups. Missing data mechanisms will be explored, and sensitivity analyses will be conducted to assess the robustness of the previously described analyses. Methods employed for sensitivity analyses may include multiple imputation or inverse probability weighting methodology [28,29].

We will evaluate secondary outcomes evaluated at 12 and 24 weeks, including efficacy outcomes, additional treatments for FI, adherence to study treatment and adverse events, comparing loperamide vs. placebo, anal sphincter exercises with biofeedback vs. usual care (educational pamphlet), and combination therapy vs. each individual treatment. For categorical outcomes, generalized linear modeling will be used instead of linear regression. We will evaluate changes from baseline to 12 and 24 weeks in condition-specific and generalized quality of life, sexual function, and adaptation comparing loperamide vs. placebo, and anal sphincter exercises with biofeedback vs. usual care (educational pamphlet), and combination therapy versus each individual treatment. Patient satisfaction with treatment modality at 12 and 24 weeks, defined as "much better" or "very much better" on the PGI-I, will be assessed using generalized linear modeling based on the predictors described in the model for the primary aims.

We will conduct exploratory analyses to evaluate whether a number of factors act as confounders (mediators) of any treatment effects for either drug/placebo or biofeedback/usual care treatments, whether these factors potentially act as effect modifiers for these treatments, or whether the factors act as independent predictors of changes in FI. This analysis will use similar models to those described for the primary outcome to model change from baseline to 12 and 24 weeks in St. Mark's (Vaizey) Score as a function of both categorical measures (presence of IBS, stool consistency, a three-level measure of treatment adherence for both treatment modalities, and adverse events) and continuous measures (bowel diary measures, constipation symptoms, digital rectal tone, anal manometry measures, and dietary fiber intake). To evaluate whether the factor acts as a confounder (mediator) of the treatment effect, the model will include the same terms as the primary outcome models with the factor added to determine whether the addition of the factor results in a change in the estimated treatment effect. To assess whether each factor acts as a treatment effect modifier, terms for the factor, the factor by treatment interaction and for the treatment by time by factor interaction will be added to the model.

A decision-analytic model will be constructed to evaluate the costeffectiveness of loperamide, anal sphincter exercises with biofeedback, and combined therapy. The analysis will be conducted from a patient and societal perspective and the model will include costs for the intervention, as well as for the management of adverse events, productivity loss and use of FI products (e.g., pad use) during the six-month period following initiation of treatment. The intervention cost will be estimated based on the amount of medication and number of exercise/biofeedback sessions that the participants used/attended during the trial. The probability of adverse events, amount of productivity loss, and FI product use will also be based on results from the CAPABLe trial. Effectiveness will be measured using quality adjusted life years (QALY). A validated algorithm developed by Brazier and Roberts (2002) will be used to generate a preference-based index score and hence QALYs based on patients' responses to the SF-12 questionnaire collected during the CAPABLe trial [30]. Compared to other utility elicitation methods (e.g., standard gamble, time trade-off), this approach helps minimize subject burden. Cost-effectiveness will be assessed by the incremental cost effectiveness ratio (ICER), which reflects the incremental cost associated with each additional QALY. In addition, we will perform sensitivity analyses to assess how the cost-effectiveness of each intervention may change when varying the value of key input parameters in the model.

Validity of anal manometry will be assessed by measuring the association between manometry measures and digital squeeze strength, incontinence severity (St. Mark's Score), global impression of improvement, and impact on quality of life as measured by the CRADI subscale on the PFDI, CRAIQ subscale on the PFIQ Modified Manchester, and FI adaptation index. Chi-square tests will be used to compare drug treatment groups with respect to the percent of participants/coordinators who responded that they thought the participant was assigned the active or placebo treatment or did not know which treatment had been assigned. Confidence intervals for the number of unmasked participants/coordinators in each treatment group will be estimated. Openended responses regarding the reason for thinking the patient was in either the active or placebo group will be categorized and reported descriptively.

\subsection{Discussion (1122/1500 words)}

This large multisite study tests two conservative treatments of FI, the antidiarrheal medication loperamide and anal sphincter exercises with biofeedback. These two treatments are recommended by clinical 
practice guidelines as first-line treatments for FI, but the evidence for their efficacy is insufficient. Studies supporting the use of loperamide for FI were small, uncontrolled studies published more than 10 years ago, and limited to patients with diarrhea-associated FI [16]. Most studies of pelvic floor biofeedback for the treatment of FI were single-site studies and have yielded inconsistent results, raising concerns that the outcomes are highly dependent on the skills and experience of the interventionist [21]. Thus, a well-controlled, multisite study to assess the efficacy of each of these treatment approaches is needed. The CAPABLe trial is the first adequately powered multi-center clinical trial to evaluate both of these primary interventions for FI, and the results will fill important voids in our knowledge of the treatment of this troubling condition.

A strength of this study is that these two treatments are being tested simultaneously in a factorial design; each active treatment, loperamide and biofeedback, is paired with an appropriate control, with loperamide being compared to placebo tablets and biofeedback compared to a standard-care educational intervention. This is an efficient study design that makes it possible to evaluate efficacy of these two independent treatments simultaneously without the cost and effort that would be required for two independent, parallel group studies. Another advantage of this design is that it will allow us to test whether combining the two active treatments is more effective than either treatment used alone. However, our target sample size provides only $55 \%$ power to detect the hypothesized 3-point difference in change from baseline in St. Mark's (Vaizey) score at 24 weeks between those randomized to both versus only one active treatment.

Loperamide is a logical choice for first-line treatment of FI because (a) diarrhea is consistently found to be the strongest risk factor for FI in population-based surveys $[1,4]$ and (b) incontinence for liquid stools is 4 times more common than incontinence for solid stools [4]. However, constipation is an adverse event that affects an estimated $2.4 \%$ of patients who are treated with loperamide for diarrhea and/or diarrhea related FI, [31] and currently loperamide is only approved by the FDA for the treatment of diarrhea. In this study, we decided not to limit enrollment to patients with diarrhea-associated FI but to include patients with normal Bristol Stool consistency ratings of 2-6 [15]. We believe the decision to include patients with a range of stool consistency provides the best opportunity to assess the generalizability of loperamide as a first-line treatment for FI. We will be able to investigate whether Bristol Stool Scale scores at enrollment predict the response to loperamide treatment in secondary analyses.

It can be challenging to recruit patients to participate in a study of the efficacy of drugs that are already available to the patient through prescription or as an over-the-counter medication; they may have a negative expectation of benefit based on their prior experience with the drug, or they may opt to try the drug without the burden of participating in the trial. The use of a factorial design may mitigate this problem because each patient is offered two treatments and has the possibility of experiencing two effective treatments in combination. In this study, we will exclude participants who have taken loperamide in the previous 30 days or who have failed a treatment trial of loperamide or diphenoxylate within the last 3 months. However, we will not eliminate all patients who have taken loperamide in the past because patients who have never taken this popular treatment for FI would likely represent a biased subset of patients who have milder symptoms of FI and/or who have constipation-related FI.

Subjects vary in their response to any investigational drug and their tolerance for its side-effects. Investigators frequently want to take this variability into account by titrating the dose of the investigational drug treatment (and the placebo) for each subject; however drug titration creates opportunities for (1) unmasking the trial and (2) introducing experimenter bias. In this study, we eliminate these threats by titrating the drug dose based on subject-ratings of improvement in FI frequency and subject-ratings of side-effects in a standardized fashion.
One challenge in designing this study was deciding on what the control group would be to compare to the anal sphincter exercises with biofeedback group. We decided to compare 6 sessions of anal sphincter exercises with biofeedback to a standard educational intervention in the form of an educational brochure delivered only at the initial visit. It could be argued that these two treatments are not balanced for nonspecific treatment components such as expectation of benefit or contact time with an interventionist. We decided to use a standard care educational control for the following reasons: (1) Biofeedback training is a complex, multicomponent treatment, and it remains difficult to identify the key mechanism(s) mediating treatment efficacy. Parallel group treatment trials often aim to isolate the key mediator of treatment efficacy, but that is not possible with biofeedback. (2) The use of a complex control condition such as 6 sessions of anal sphincter exercises taught without biofeedback devices would introduce additional non-specific treatment components, such as targeted counseling from the interventionist that would make it difficult to interpret the loperamide vs. placebo arm of the study in the context of this factorial design. For these reasons, we concluded that we should first establish that anal sphincter exercises with biofeedback are superior to minimum standard care (e.g. education) in an adequately controlled multisite study.

Two challenges in evaluating behavioral interventions such as biofeedback are (1) that the intervention protocol is not standardized so there are variations in how it is implemented in different clinics, and (2) the outcomes seem to depend on the skill and experience of the interventionists. To address the first problem, a group of experts on the biofeedback treatment of FI worked to disaggregate biofeedback training for FI into three key procedures: strength training, sensory discrimination training to improve the detection of rectal filling, and desensitization to the sensation of urgency to defecate. We then collaborated with a device manufacturer to develop separate software programs for each of these biofeedback procedures. To address the second problem, the protocol was standardized in the manual of operations, required in person training and certification, and monitored through audiotapes.

The design, interventions and outcome measures of the CAPABLe trial have been carefully considered in order to provide valid, reliable estimates of the efficacy, safety and cost-effectiveness of two commonly used primary conservative therapies for women with FI with a focus on patient-centered outcomes. This study will provide important foundational evidence for the treatment of this common and burdensome condition.

\section{Appendix A. Pelvic floor disorders network contributors}

\section{Cleveland Clinic}

J. Eric Jelovsek, Principal Investigator

Mathew D. Barber, Co-investigator

Marie Fidela R. Paraiso, Co-investigator

Mark D. Walters, Co-investigator

Beri Ridgeway, Co-investigator

Brooke Gurland, Co-investigator

Massarat Zutshi, Co-investigator

Geetha Krishnan, Research Nurse

Ly Pung, Research Nurse

Annette Graham, Research Nurse

Alpert Medical School of Brown University-Women \& Infant's

\section{Hospital of Rhode Island}

Vivian W. Sung, Principal Investigator

Deborah L. Myers, Co-investigator

Charles R. Rardin, Co-investigator

Cassandra Carberry, Co-investigator

B. Star Hampton, Co-investigator

Kyle Wohlrab, Co-investigator

Ann S. Meers, RN, Research Nurse 


\section{Duke University}

Anthony Visco, Principal Investigator

Cindy Amundsen, Co-investigator

Alison Weidner, Co-investigator

Nazema Siddiqui, Co-investigator

Amie Kawasaski, Co-investigator

Shantae McLean, Clinical Site Coordinator

Nicole Longoria, Clinical Research Coordinator

Jessica Carrington, Clinical Research Coordinator

Niti Mehta, Clinical Research Specialist

Ingrid Harm-Ernandes, Interventionist

Jennifer Maddocks, Interventionist

Amy Pannullo, Interventionist

University of Alabama at Birmingham

Alayne Markland, Primary Investigator

Holly Richter, Co-investigator

R. Edward Varner, Co-investigator

Robert Holley, Co-investigator

L. Keith Lloyd, Co-investigator

Tracy S. Wilson, Co-investigator

Alicia Ballard, Co-investigator

Jeannine McCormick, Research Nurse

Velria Willis, Research Nurse

Nancy Saxon, Research Nurse

Kathy Carter, Research Nurse

Julie Burge, Research Coordinator

NIH Project Scientist

Susan Meikle, Co-investigator

University of California, San Diego

Charles Nager, Principal Investigator

Michael Albo, Co-investigator

Emily Lukacz, Co-investigator

Cindy Furey, Interventionist

Patricia Riley, Interventionist

JoAnn Columbo, Research Coordinator

Sherella Johnson, Research Coordinator

Kaiser Permanente - San Diego

Shawn Menefee, Co-investigator

Karl Luber, Co-investigator

Keisha Dyer, Co-investigator

Gouri Diwadkar, Co-investigator

Jasmine Tan-Kim, Co-investigator

University of New Mexico

Rebecca G. Rogers, Primary Investigator

Yuko Komesu, Co-investigator

Gena Dunivan, Co-investigator

Peter Jeppson, Co-investigator

Sara Cichowski, Co-investigator

Christy Miller, Interventionist

Erin Yane, Interventionist

Julia Middendorf, Research Nurse

Risela Nava, Research Coordinator

RTI

Dennis Wallace, Principal Investigator

Marie G. Gantz, Alternate Principal Investigator

Amanda Youmans-Weisbuch, Clinical Study Specialist

Poonam Pande, Chemistry, Manufacturing, and Controls Project

Leader

Kelly Roney, Regulatory Project Leader

Ryan E. Whitworth, Statistician

Lauren Klein Warren, Statistician

Kevin A. Wilson, Clinical Research Informatics Project Leader

Daryl Matthews, Data Manager

James W. Pickett, II, Programmer

Yan Tang, Programmer

Tamara L. Terry, Research Manager
Jutta Thornberry, Program Manager

Kristin Zaterka-Baxter, Clinical Study Specialist

Lindsay Morris, Research Coordinator

Amanda Honeycutt, Economist

University of Pennsylvania

Lily Arya, Principal Investigator

Ariana Smith, Co-investigator

Heidi Harve, Co-investigator

Uduak Umoh Andy, Co-investigator

Pamela Levin, Co-investigator

Diane K. Newman, Co-investigator

Mary Wang, Interventionist

Donna Thompson, Interventionist

Teresa Carney, Interventionist

Michelle Kingslee, Research Coordinator

Lorraine Flick, Research Nurse

University of Pittsburgh

Halina Zyczynski, Principle Investigator

Pam Moalli, Co-investigator

Gary Sutkin, Co-investigator

Jonathan Shepherd, Co-investigator

Michael Bonidie, Co-investigator

Steven Abo, Co-investigator

Janet Harrison, Co-investigator

Lori Geraci, Research Coordinator

Judy Gruss, Research Coordinator

Karen Mislanovich, Research Coordinator

Ellen Eline, Interventionist

Beth Klump, Interventionist

University of North Carolina at Chapel Hill

William E. Whitehead Ph.D., Co-investigator

\section{References}

[1] R.L. Nelson, Epidemiology of fecal incontinence, Gastroenterology 126 (2004) S3-S7.

[2] J.E. Jelovsek, M.D. Barber, M.F. Paraiso, M.D. Walters, Functional bowel and anorectal disorders in patients with pelvic organ prolapse and incontinence, Am. J. Obstet. Gynecol. 193 (2005) 2105-2111.

[3] Y. Gorina, S. Schappert, A. Bercovitz, N. Elgaddal, E. Kramarow, Prevalence of incontinence among older Americans, Vital Health Stat. 3 (36) (2014) 1-33.

[4] W.E. Whitehead, L. Borrud, P.S. Goode, S. Meikle, E.R. Mueller, A. Tuteja, et al., Fecal incontinence in US adults: epidemiology and risk factors, Gastroenterology 137 (2009) $(512,7,517 . e 1-2)$

[5] W.E. Whitehead, S.S. Rao, A. Lowry, D. Nagle, M. Varma, K.N. Bitar, et al., Treatment of fecal incontinence: state of the science summary for the National Institute of Diabetes and Digestive and Kidney Diseases Workshop, Am. J. Gastroenterol. 110 (1) (2014) 138-346

[6] S. Heymen, Y. Scarlett, K. Jones, Y. Ringel, D. Drossman, W.E. Whitehead, Randomized controlled trial shows biofeedback to be superior to pelvic floor exercises for fecal incontinence, Dis. Colon Rectum 52 (2009) 1730-1737.

[7] C. Norton, W.E. Whitehead, D.Z. Bliss, P. Metsola, J. Tries, Conservative and pharmacological management of faecal incontinence in adults, in: P. Abrams, L. Cardozo, S. Khoury, A. Wein (Eds.), Incontinence, Health Publications, Plymouth 2005. pp. 1521-1563.

[8] C.J. Vaizey, E. Carapeti, J.A. Cahill, M.A. Kamm, Prospective comparison of faecal incontinence grading systems, Gut 44 (1999) 77-80.

[9] T. Hallgren, S. Fasth, D.S. Delbro, S. Nordgren, T. Oresland, L. Hulten, Loperamide improves anal sphincter function and continence after restorative proctocolectomy, Dig. Dis. Sci. 39 (1994) 2612-2618.

[10] W.M. Sun, N.W. Read, M. Verlinden, Effects of loperamide oxide on gastrointestinal transit time and anorectal function in patients with chronic diarrhoea and faecal incontinence, Scand. J. Gastroenterol. 32 (1997) 34-38.

[11] L.R. Schiller, C.A. Santa Ana, S.G. Morawski, J.S. Fordtran, Mechanism of the antidiarrheal effect of loperamide, Gastroenterology 86 (1984) 1475-1480.

[12] M. Read, N.W. Read, D.C. Barber, H.L. Duthie, Effects of loperamide on anal sphincter function in patients complaining of chronic diarrhea with fecal incontinence and urgency, Dig. Dis. Sci. 27 (1982) 807-814.

[13] F. Herbst, M.A. Kamm, R.J. Nicholls, Effects of loperamide on ileoanal pouch function, Br. J. Surg. 85 (1998) 1428-1432.

[14] M. Fox, B. Stutz, D. Menne, M. Fried, W. Schwizer, M. Thumshirn, The effects of loperamide on continence problems and anorectal function in obese subjects taking orlistat, Dig. Dis. Sci. 50 (2005) 1576-1583.

[15] S.J. Lewis, K.W. Heaton, Stool form scale as a useful guide to intestinal transit time, Scand. J. Gastroenterol. 32 (1997) 920-924. 
[16] M.I. Omar, C.E. Alexander, Drug treatment for faecal incontinence in adults, Cochrane Database Syst. Rev. 6 (2013), CD002116.

[20] M.J. Atkinson, A. Sinha, S.L. Hass, S.S. Colman, R.N. Kumar, M. Brod, et al., Validation of a general measure of treatment satisfaction, the Treatment Satisfaction Questionnaire for Medication (TSQM), using a national panel study of chronic disease, Health Qual. Life Outcomes 2 (2004) 12.

[21] C. Norton, J.D. Cody, Biofeedback and/or sphincter exercises for the treatment of faecal incontinence in adults, Cochrane Database Syst. Rev. 7 (2012), CD002111.

[23] J.H. Wagner, A.C. Justice, M. Chesney, G. Sinclair, S. Weissman, M. RodriguezBarradas, et al., Patient- and provider-reported adherence: toward a clinically useful approach to measuring antiretroviral adherence, J. Clin. Epidemiol. 54 (Suppl. 1) (2001) S91-S98.

[24] J.C. Walsh, S. Mandalia, B.G. Gazzard, Responses to a 1 month self-report on adherence to antiretroviral therapy are consistent with electronic data and virological treatment outcome, AIDS 16 (2002) 269-277.

[25] L. Osterberg, T. Blaschke, Adherence to medication, N. Engl. J. Med. 353 (2005) 487-497.

[26] M. Deutekom, M.P. Terra, A.C. Dobben, M.G. Dijkgraaf, R.J. Felt-Bersma, J. Stoker, et al., Selecting an outcome measure for evaluating treatment in fecal incontinence, Dis. Colon Rectum 48 (2005) 2294-2301.

[27] E.M. Bols, E.J. Hendriks, M. Deutekom, B.C. Berghmans, C.G. Baeten, R.A. de Bie, Inconclusive psychometric properties of the Vaizey score in fecally incontinent patients: a prospective cohort study, Neurourol. Urodyn. 29 (2010) 370-377.

[28] R. Little, R. Rubin (Eds.), Statistical Analysis With Missing Data, 2nd ed.Wiley Interscience, Hoboken, NJ, 2002.

[29] J.M. Robins, A. Rotnitzky, L.P. Zhao, Analysis of semiparametric regression models for repeated outcomes in the presence of missing data, J. Am. Stat. Assoc. 90 (1995) 106-129.

[30] J.E. Brazier, J. Roberts, The estimation of a preference-based measure of health from the SF-12, Med. Care 42 (2004) 851-859.

[31] B. Meuris, Observational study of travelers' diarrhea, J. Travel Med. 2 (1995) 11-15.
[32] R.G. Rogers, T.H. Rockwood, M.L. Constantine, R. Thakar, D.N. Kammerer-Doak, R.N Pauls, et al., A new measure of sexual function in women with pelvic floor disorders (PFD): the Pelvic Organ Prolapse/Incontinence Sexual Questionnaire, IUGA-Revised (PISQ-IR), Int. Urogynecol. J. 24 (2013) 1091-1103.

[33] J.E. Jelovsek, M.D. Barber, Women seeking treatment for advanced pelvic organ prolapse have decreased body image and quality of life, Am. J. Obstet. Gynecol. 194 (2006) 1455-1461.

[34] P. Hopwood, I. Fletcher, A. Lee, S. Al Ghazal, A body image scale for use with cancer patients, Eur. J. Cancer 37 (2001) 189-197.

[35] G. Block, C. Gillespie, E.H. Rosenbaum, C. Jenson, A rapid food screener to assess fat and fruit and vegetable intake, Am. J. Prev. Med. 18 (2000) 284-288.

[36] G. Block, A.M. Hartman, C.M. Dresser, M.D. Carroll, J. Gannon, L. Gardner, A databased approach to diet questionnaire design and testing, Am. J. Epidemiol. 124 (1986) 453-469.

[37] G.F. Longstreth, W.G. Thompson, W.D. Chey, L.A. Houghton, F. Mearin, R.C. Spiller, Functional bowel disorders, Gastroenterology 130 (2006) 1480-1491.

[38] M.D. Barber, M.D. Walters, R.C. Bump, Short forms of two condition-specific qualityof-life questionnaires for women with pelvic floor disorders (PFDI-20 and PFIQ-7), Am. J. Obstet. Gynecol. 193 (2005) 103-113.

[39] S. Kwon, A.G. Visco, M.P. Fitzgerald, W. Ye, W.E. Whitehead, Pelvic Floor Disorders Network (PFDN). Validity and reliability of the Modified Manchester Health Questionnaire in assessing patients with fecal incontinence, Dis. Colon Rectum 48 (2005) 323-331 (discussion 331-4).

[40] J. Ware Jr., M. Kosinski, S.D. Keller, A 12-item short-form health survey: construction of scales and preliminary tests of reliability and validity, Med. Care 34 (1996) 220-233.

[41] L. Frank, L. Kleinman, C. Farup, L. Taylor, P. Miner Jr., Psychometric validation of a constipation symptom assessment questionnaire, Scand. J. Gastroenterol. 34 (1999) 870-877.

[42] I. Yalcin, R.C. Bump, Validation of two global impression questionnaires for incontinence, Am. J. Obstet. Gynecol. 189 (1) (Jul 2003) 98-101. 